\title{
A critical appraisal of the recommendations for the use of meningococcal conjugate vaccines
}

\author{
Marco Aurélio Palazzi Sáfadi, ${ }^{1}$ Eitan Naaman Berezin, ${ }^{2}$ Gabriel Wolf Oselka ${ }^{3}$
}

\begin{abstract}
Objectives: To assess the epidemiology of meningococcal disease (MD) in Brazil and the impact that recent evidence and lessons learned from the introduction of meningococcal C conjugate (MCC) vaccines into immunization programs may have on different strategies of vaccine use.

Sources: Non-systematic review of the MEDLINE, SciELO and LILACS databases covering the period from 2000 to 2011.

Summary of the findings: Meningococcal disease is endemic in Brazil, with periodic occurrence of outbreaks. Most cases are associated with serogroup $C$ and the highest incidence rates are observed in infants, encouraging the introduction of MCC vaccine in the National Immunization Program in 2010 for children under 2 years old. The introduction of MCC vaccines into immunization programs in Europe, Canada and Australia proved to be effective, with dramatic reduction in the incidence of serogroup $C$ meningococcal disease, not only in the vaccinated, but also in the unvaccinated individuals. Long-term effectiveness of MCC vaccines was dependent on a combination of antibody persistence, immunologic memory and herd protection. Recent evidence indicating that antibody persistence is not long-lasting in young immunized children, and that immunologic memory is not fast enough to protect them against the disease, emphasize the importance of herd protection to maintain the population protected.

Conclusions: The rapid decline of antibody titers in children vaccinated in the first years of life suggests the need to incorporate booster doses before adolescence, especially in locations like Brazil, where the immunization program did not incorporate catch-up campaigns including adolescents, lacking the herd immunity effect.
\end{abstract}

J Pediatr (Rio J). 2012;88(3):195-202: Neisseria meningitidis, meningococcal disease, conjugate meningococcal vaccines.

\section{Introduction}

Meningococcus (Neisseria meningitidis) is currently the leading cause of bacterial meningitis in Brazil. ${ }^{1}$ The invasive infection by Neisseria meningitidis results in a wide spectrum of clinical disease that includes meningitis, meningococcemia, or both, with the former being the most frequently observed clinical form. Some of the characteristics of meningococcal disease, as its rapid onset, severity and high fatality rates, as well as its potential epidemic nature, make the possibility of prevention of this infection, through vaccination, extremely important. ${ }^{2}$

The licensing of MCC, from the late 1990s, represented a huge advance in the ability to control serogroup $\mathrm{C}$ meningococcal disease. Different strategies of routine immunization were used in the introduction of these vaccines in several countries in Europe, Canada and Australia, all of them accompanied by a dramatic reduction in incidence of serogroup $\mathrm{C}$ meningococcal disease, with success in controlling the disease shortly after its introduction. ${ }^{2-10}$

One of the most important lessons learned with the introduction of MCC vaccines in Europe was that these vaccines not only provided direct protection against vaccinated individuals, but also reduced the disease among unvaccinated individuals, an effect attributed to its ability to prevent the acquisition of the nasopharynx carrier state of

1. PhD. Assistant professor, Departamento de Pediatria, Faculdade de Ciências Médicas da Santa Casa de São Paulo (FCMSCSP), São Paulo, SP, Brazil. 2. PhD. Adjunct professor, Departamento de Pediatria, FCMSCSP, São Paulo, SP, Brazil.

3. PhD. Associate professor, Departamento de Pediatria, Faculdade de Medicina, Universidade de São Paulo (USP), São Paulo, SP, Brazil.

Conflicts of interest: M.A.P.S. has received lecture and/or consulting feeds from Baxter, GSK, Novartis, Sanofi, and Wyeth/Pfizer. G.W.O. has received lecture and/or consulting feeds from Baxter, Novartis, Sanofi, and Wyeth/Pfizer. E.N.B. has received lecture and/or consulting feeds from Baxter, Novartis, and Wyeth/Pfizer.

Suggested citation: Sáfadi MA, Berezin EN, Oselka GW. A critical appraisal of the recommendations for the use of meningococcal conjugate vaccines. J Pediatr (Rio J). 2012;88(3):195-202.

Manuscript submitted Oct 16 2011, accepted for publication Nov 162011.

http://dx.doi.org/10.2223/JPED.2167 
the of meningococcus $\mathrm{C}$ among the vaccinated, causing the circulation and the spread of the bacteria to diminish in the population in general, reducing, thus, the risk of infection. Furthermore, it was found that the persistence of serum bactericidal antibody (SBA) titers associated to protection are essential to maintain the individual protection against the disease, being more important than the presence of the immunologic memory, often insufficient to guarantee, alone, the protection against an invasive disease, of short incubation period. 10

This article presents a summary of the epidemiological characteristics of meningococcal disease in Brazil and discusses the effect of the experience gained from Europe, Canada and Australia, with the use of MCC vaccines, on the strategies for prevention of meningococcal disease.

\section{Etiopathogenesis and epidemiology}

The causative agent of meningococcal disease, Neisseria meningitidis, is a gram-negative, aerobic, non-mobile diplococcus, belonging to the Neisseriaceae family. The antigenic composition of the polysaccharide capsule enables the classification of $N$. Meningitis into 13 different serogroups: A, B, C, D, H, I, K, L, W135, X, Y, Z and 29E. Currently, serogroups $A, B, C, Y, W 135$ and $X$ are responsible for nearly all cases of disease infecting only humans. Meningococci are also classified into serotypes and serosubtypes, according to the antigenic composition of the outer membrane proteins PorB and PorA, respectively. ${ }^{2}$ Meningococci have demonstrated the ability to exchange the genetic material that is responsible for producing the capsule and, thereby, to change the serogroup $B$ to $C$ or vice-versa. Because the protection granted by the vaccines is serogroup-specific, this phenomenon may have consequences for the formulation and use of anti-meningococcal vaccines. Genetic multilocus sequence typing, based on polymorphisms in multiple genes, is currently used to monitor the global epidemiology of meningococcal disease, making it possible to distinguish the capsular switching between meningococcal strains. ${ }^{2}$

The invasive infection by Neisseria meningitidis results in wide spectrum of clinical disease, which includes meningitis and meningococcemia, or both, with the former being the most frequently observed clinical form. ${ }^{5}$ The name meningococcal disease becomes suitable in this context, being adopted internationally.

Meningococcus infects and colonizes only the human nasopharynx, and is spread from person to person through contact with respiratory secretions (e.g., while sharing a drink in the same cup, through kissing, etc.) or by inhalation of aerosol droplets. The asymptomatic nasopharyngeal colonization by Neisseria meningitides characterizes the carrier state, and it is the focus from which the bacteria can be transmitted. The carrier state occurs frequently, and may be more than $10 \%$ in determined age groups during endemic periods, and the individual may carry the meningococcus for a long period. 2,5,7 The incidence rates of carriage are low in the first years of life, increasing among adolescents and young adults and in less privileged social classes. In the great majority of these individuals, asymptomatic nasopharyngeal colonization by typable and non-typable Meningococci and by other species of Neisseria - such as, for instance, the Neisseria lactamica - ends up functioning as an immunizing process, resulting in the production of protective antibodies. ${ }^{2,11}$

After the colonization of the nasopharynx, the chance of developing the disease will depend on the virulence of the strain, on the immune status of the host, and on the ability to eliminate the agent from the bloodstream through serum antibodies with bactericidal activity mediated by the activation of the complement, resulting in opsonization and lysis of the bacteria. The spleen also plays an important role in the elimination of the bacteria from the bloodstream. Goldschneider et al. demonstrated, in the 1960s, in American soldiers, the direct correlation between susceptibility to invasive meningococcal disease and the absence of detectable serum bactericidal antibodies. ${ }^{12}$

The invasive meningococcal disease occurs primarily in susceptible people recently colonized by a pathogenic strain. Numerous risk factors have been associated to meningococcal disease, such as: recent viral respiratory infections (especially influenza), household agglomerations, living in barracks or camps for students, smoking (passive or active), less privileged socioeconomic conditions and close contact with patients. The risk of developing invasive disease in close contacts of a patient is about 500 to 800 times higher than in the population in general.5,7 The following conditions are also associated to higher risk of meningococcal disease: asplenia (anatomic or functional), deficiency of properdin, C3 and of terminal components of the complement ( $\mathrm{C} 5$ to $\mathrm{C} 9$ ) that, due to the inability to cause intracellular bacterial death, are at higher risk of recurrent episodes of meningococcal disease, considered, therefore, priority groups for prophylactic vaccination. 2,13

It is estimated that approximately 500,000 cases of invasive meningococcal disease occur every year worldwide, leaving about 60,000 patients with permanent sequelae and resulting in over 50,000 deaths. ${ }^{14}$

Meningococcal disease occurs worldwide; there are, however, marked geographical differences in incidence and in the distribution of serogroups that cause the disease. Historically, serogroup A is associated with endemic disease in developing countries, especially in Sub-Saharan Africa, known as the meningitis belt. The annual incidence of the disease, during these epidemics, can reach rates as high as 1,200 cases per 100,000 inhabitants. Recently, outbreaks of the serogroup $X$ disease were reported in Niger, Togo, Kenya and Ghana, besides outbreaks of serogroup W135, belonging to the clonal complex ST-11, 
reported in Muslim pilgrims in Saudi Arabia and also in countries of the African belt. ${ }^{14,15}$

In developed countries of North America and Europe, the occurrence of the disease is endemic. In Europe, more than $95 \%$ of cases were attributed to serogroups B and C. From the late 1990s, some countries, such as the United Kingdom, Ireland, Spain, Portugal, the Netherlands, Belgium and Iceland, observed an increase in incidence rates of cases attributed to serogroup C, related to the hypervirulent ST11 clonal complex, encouraging the introduction of routine immunization programs with the MCC vaccine. These countries observed a dramatic reduction in the incidence of serogroup $C$ meningococcal disease immediately after the introduction of the MCC vaccines. $6,10,16$ In the U.S., the incidence rates of meningococcal disease have been declining $n$ recent years, with serogroups $B, C$ and $Y$ responsible, each, for about $1 / 3$ of reported cases. ${ }^{14,17}$

In Brazil, meningococcal disease is endemic, with periodic outbreaks in several municipalities. Incidence rates have been stable in recent years, with approximately 1.5-2 cases per 100,000 inhabitants. ${ }^{18-20}$ Due to the large proportion of meningitis notified without identification of the etiological agent, the real incidence of the disease in our environment should probably be higher than routinely reported. The recent publication of the results of a study in sentinel hospitals in the state of Sao Paulo, 19 with the use of molecular biology techniques (real-time polymerase chain reaction, RT-PCR) to search for Streptococcus pneumoniae, Neisseria meningitidis and Haemophilus influenzae $b$ in blood and cerebrospinal fluid (CSF) samples collected from patients diagnosed with meningitis, showed an increase of 52, 85 and $20 \%$, respectively, in the identification of these agents in comparison to the conventional methods of culture. These findings emphasize the need to broaden the availability of these molecular biology techniques to other locations, so we can have a more representative scenario of the real load of the meningococcal disease in our country.

Meningococcal disease affects individuals from all age groups, but approximately $40-50 \%$ of cases reported in Brazil occur in children under 5 years old, with the highest incidence rates of disease consistently observed in infants in the first year of life. ${ }^{18}$ Another important feature observed in Brazil is the absence, in endemic periods, of peaks in adolescents, in contrast to what is observed in European and North American countries.

The case fatality rates of the disease in our country, are, unfortunately, quite high, standing at around 18 to $20 \%$ in recent years, despite all the improvements we have achieved in health care in this period. 18,20,21 When meningococcemia is assessed, case fatality rate is as high as almost $50 \%$, showing the importance of discussing strategies to prevent the disease. Regarding the distribution of cases according to serogroups, a significant increase has been observed in the number and proportion of cases attributed to serogroup $\mathrm{C}$ in different regions of the country in recent years, such as in the states of Bahia, Pernambuco, Rio de Janeiro, Minas Gerais and São Paulo, making it currently the main cause of meningococcal disease in the country. ${ }^{20}$ In 2010, in the state of São Paulo, which has the highest incidence rates of meningococcal disease reported in the country (3.5 cases per 100,000 inhabitants), serogroup C accounted for $81.5 \%$ of the cases identified; serogroup $B$, for $10.9 \%$; serogroup W135, for 6\%; and the serogroup $Y$, by $1.2 \% .21$

An outbreak is defined from the occurrence of three or more cases, confirmed or probable, in a period of three months, in the same geographical area, generating an attack rate greater than or equal to 10 cases per 100,000 inhabitants. ${ }^{18}$ Epidemics caused by Neisseria meningitides are characterized by a predominance of a meningococcus genotype, high incidence rates and an increase in incidence in older age groups. Brazil has reported, in recent years, various outbreaks caused by serogroup $\mathrm{C}^{22}$ associated to the clonal complex ST-103, in different regions of the country, which confirms this clonal complex as being responsible for the increase in incidence rates of the serogroup $\mathrm{C}$ meningococcal disease. ${ }^{23}$

Another phenomenon that highlights the unpredictable behavior of the meningococcus is the recent finding of the emergence of serogroup W135 as a cause of meningococcal disease in the Southern region of the country, ${ }^{24}$ as well as in São Paulo and Rio de Janeiro. ${ }^{25,26}$ The strains of serogroup W135, antigenically characterized, belonged mostly to the clonal complex ST-11, which is the same responsible for the epidemics of serogroup W135 in Muslim pilgrims in 2000 and 2001.

\section{Development of meningococcal conjugate vaccines}

The polysaccharide vaccines, available in Brazil for about 40 years, provide protection for serogroups A and C. Such vaccines, as with other non-conjugated polysaccharide vaccines, do not generate adequate immune response in children under 2 years old due to the lack of consistent response to T-independent antigens in this age group. Another important characteristic of these vaccines is that, even in children over 2 years old, the protection provided is of limited duration, and is not able to induce immunologic memory. They present, still, the possibility to induce hyporesponsiveness in subsequent doses. These vaccines, thus, are not routinely used and are only indicated for highrisk groups or in the control of outbreaks or epidemics $2,6,7,9$ because of all their characteristics, combined with the fact that they exert only transitory and incomplete effect in reducing the colonization and the transmission of the meningococci in the vaccinated population.

The conjugation of polysaccharides with carrier proteins (nontoxic mutant diphtheria toxin - CRM197 - or tetanus 
toxoid), alters the nature of the anti-polysaccharide response to a $\mathrm{T}$ - dependent response. B cells, by recognizing the polysaccharide, process the conjugate protein carrier and present the peptide epitopes to the T-CD4+ cells. This antigenic complex induces the production of high levels of antibodies (even in young infants), higher avidity of antibodies, higher serum bacterial activity and, still, the formation of populations of memory B lymphocytes, of long duration, providing an excellent anamnestic response (booster effect) in the re-exposure. Furthermore, these vaccines have the ability to reduce nasopharyngeal colonization by serogroup $\mathrm{C}$, reducing the number of carriers among the vaccinated and, therefore, the spread of the disease in the population (indirect immunity or herd protection). $2,6,7,9$

Pharmaceutical companies initially developed, in the late 1990s, monovalent meningococcal conjugate vaccines against meningococcus $\mathrm{C}$, containing one polysaccharide, conjugated to the mutant diphtheria toxin (MCC-CRM197 - Wyeth and Novartis Laboratories) or to the tetanus toxid (MCC-TT - Baxter Laboratory). These vaccines have proven to be immunogenic in infants, toddlers, older children, adolescents and adults. Later, it was also licensed, initially in the United Kingdom, a combined Haemophilus influenzae type b (Hib)-MenC vaccine conjugated to the tetanus toxoid (GSK). 2,6,9

The randomized, controlled, phase III trials, that assess the efficacy of the vaccine in a determined population, due to low incidence of the serogroup $\mathrm{C}$ meningococcal disease, are nor feasible in practice. Thus, the serologic markers of immunity against infection by meningococcal $C$ are used to infer the effectiveness of these vaccines and served as a basis for their licensing. 2,6 As previously described, the correlate of protection accepted, i.e., the lowest antibody titer necessary to consider the vaccinated individual protected, is the presence of SBA $\geq 4$ using human complement or SBA titers $\geq 8$ when using complement obtained from baby rabbits. 2,6 During the pre and post-licensure trials, excellent immunogenicity in the short-term and presence of immunologic memory associated to available conjugate vaccines were demonstrated, besides adequate tolerability and reactogenicity profiles. $2,6,9$

The vaccines were initially licensed in Europe, in 1999, with three doses for primary immunization of infants from 2 months of age. However, later immunogenicity trials showed that the scheme of primary immunization could be reduced to only two doses in this age group. The MCC vaccines were licensed in Brazil from 2001.

\section{Experience with mass immunization of the population with meningococcal $\mathrm{C}$ conjugate vaccine}

The first place to introduce the MCC vaccine as a routine schedule was the United Kingdom, in 1999, vaccinating in least than 1 year more than 15,000,000 individuals younger than 17 years. ${ }^{3}$ The initial results were encouraging, with a reduction of $81 \%$ in incidence of the serogroup C meningococcal disease from the period of 1998-1999 compared to the period of 2000-2001. The effectiveness of the vaccine was of up to $97 \%$ in adolescents and of $92 \%$ in toddlers, who received one dose of the vaccine, and of $91 \%$ in infants, who received three doses of the vaccine, at ages 2, 3 and 4 months. The number of deaths attributed to serogroup $\mathrm{C}$ meningococcal disease dropped from 67 in 1999 , to five in 2001.27 There was a significant reduction in the incidence of meningococcal disease even in unvaccinated age groups, demonstrating that the conjugate vaccines protect not only the vaccinated individuals, but also the general population, due to the reduction of the number of carriers of the bacteria in nasopharynx.28-30 (Figure 1 ). The success of the mass immunization program was, therefore, attributed both to the high effectiveness of the vaccine (direct protection) and to the herd effect (indirect protection) (Figure 1).

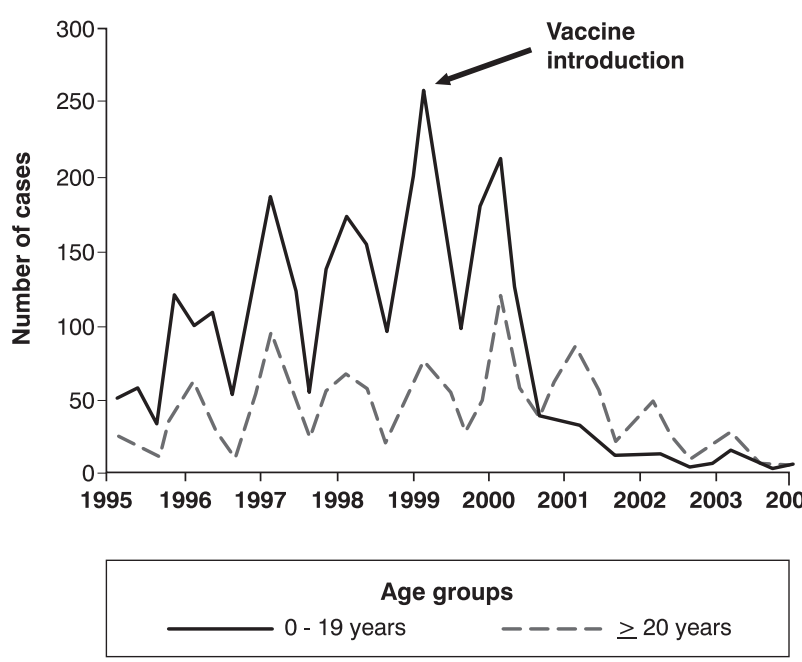

Figure 1 - Impact of the immunization program with meningococcal C conjugate vaccine on the United Kingdom. Adapted from Trotter et al. 4

However, few years after the introduction of the vaccine in the United Kingdom, from 2004, it was observed a decline in effectiveness for all age groups, especially in the group of infants which was vaccinated in the routine schedule at 2, 3 and 4 months. ${ }^{4}$ Between 2000 and 2003, 53 cases of the meningococcal $C$ disease were registered in vaccinated children, and the investigation of these cases demonstrated no evidence of immunodeficiency. A similar phenomenon was observed in Spain, with a loss of protection in children that were vaccinated at 2, 4 and 6 months of age. 30,31 
Another important observation was that, in cases of vaccine failure, meningococcal $\mathrm{C}$ disease occurred despite the presence of immunologic memory in the previously vaccinated patients, as a consequence of the disease being potentially associated to short incubation periods, highlighting the importance of persistence of protective antibody titers to maintain individual protection. ${ }^{32}$

The results of monitoring incidence of disease caused by serogroup $\mathrm{C}$, appointing for loss of efficacy of the vaccine after some years, occurring mainly in children immunized in the routine, in the first years of age, with two or three doses of the vaccine, appointed, thus, to the need for use of different routine immunization schedules from the ones so far employed. From 2006, it was then incorporated a booster dose after 1 year of age, in order to try to ensure a longer protection for infants immunized in the first years of life. 33

The effect of the mass vaccination in rates of carriers was assessed in a study in the United Kingdom involving 16,000 adolescents from 15 to 17 years, which observed a $66 \%$ reduction in rates of nasopharyngeal carriers, comparing the periods before and after the introduction of the conjugate meningococcal vaccines. ${ }^{29}$ In this same study, it was observed no alteration to other serogroups carriage rates among the vaccinated population. One of the concerns that arose after the dramatic reduction in the incidence of serogroup $\mathrm{C}$ meningococcal disease in countries that adopted mass vaccination, was the possibility of the introduction of conjugate vaccine to exert immune pressure in the population of meningococci, resulting in exchange to another serogroup - for instance, $B$ - for which there are no universally effective vaccines. Until now, surveillance data have shown that in the UK, alongside the decline in the incidence of serogroup $\mathrm{C}$ meningococcal disease, it has not been observed an increase in the absolute number of cases caused by the serogroup B. ${ }^{34}$ Doubts in relation to the real possibility of capsular shifting and exchange of the serogroup emphasize the need for a rigorous surveillance system in the places that introduced the MCC in their routine immunization schedule.

An alternative strategy was adopted in the Netherlands, which in 2002 started a routine immunization program with only one dose of the MCC vaccine conjugated to tetanus toxoid at 14 months of age. Additionally, a catchup campaign was introduced with the aim to immunize all children and adolescents from 1 to 18 years with the same vaccine. The first results showed a dramatic reduction in the incidence of meningococcal disease both in vaccinated and unvaccinated age groups, and the greatest reduction (99\%) was verified in vaccinated age groups. ${ }^{35}$ The number of reported cases dropped from 276 in 2001 to only four in 2005, and so far there are no reports of vaccine failures in the Netherlands. ${ }^{36}$ The success obtained in the United Kingdom and in the Netherlands was also verified in other European countries, where significant reductions can be seen in the incidence of serogroup $C$ meningococcal disease after the introduction of the MCC vaccines in immunization programs ${ }^{35-40}$ (Figure 2).

These vaccines were also successfully used to control outbreaks of the serogroup $C$ meningococcal disease. After years of persistence of meningococcal $C$ disease, despite the use of polysaccharide vaccines to control outbreaks, it was used in the population of Quebec, Canada, the MCC vaccine for all the individuals from 2 months to 20 years old. The vaccine effectiveness, verified more than one year after the outbreak, was of more than $96 \%$, demonstrating its potential use in controlling epidemics. ${ }^{41}$

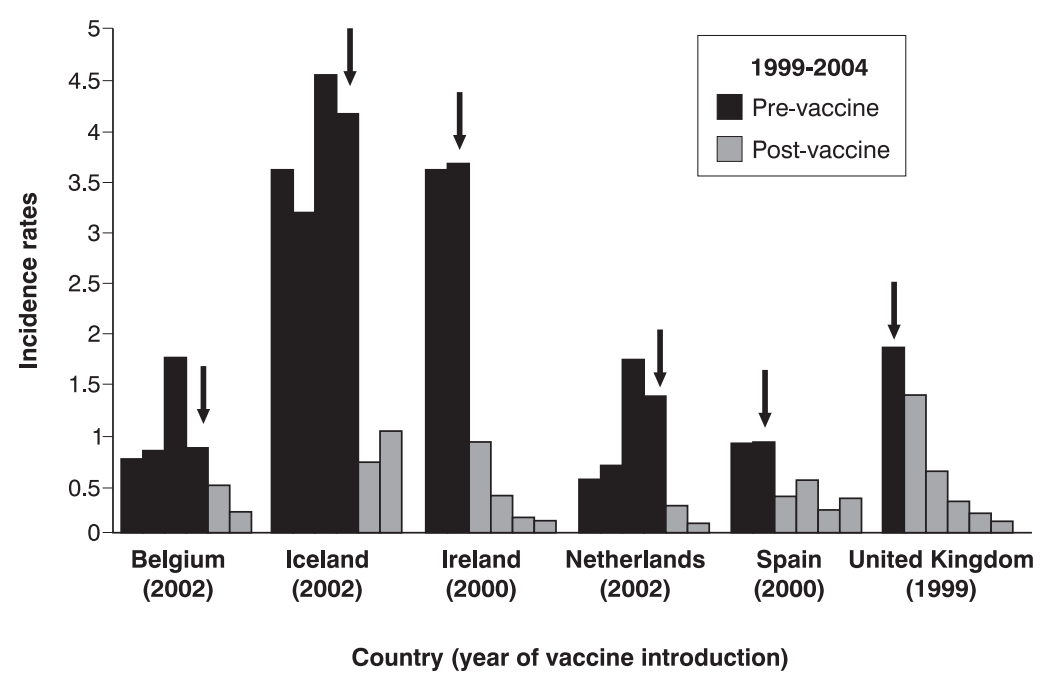

Figure 2 - Impact of the immunization programs with the MCC vaccines on different European countries. Adapted from Trotter et al. ${ }^{39}$ 
Recently, however, studies in the United Kingdom, 42 which assessed the persistence of protective antibody titers (above the correlate of protection: serum bactericidal antibody measured with rabbit complement - rSBA $\geq 8$ ) among children and adolescents vaccinated in different ages and in different schemes, showed that, among the children vaccinated between 2 months and 6 years old, only $25 \%$ had protective antibody titers 6 to 7 years after immunization. The study observed low rates of children with protective antibody titers in all age groups vaccinated before 6 years old: $12 \%$ among children who received the vaccine within 6 months of age, $16 \%$ of the children vaccinated between 5 and 11 months; $26 \%$ in the vaccinated between 1 and 2 years; $23 \%$ in the vaccinated between 2 and 3 years; $33 \%$ in the vaccinated between 3 and 4 years; $26 \%$ in the vaccinated between 4 and 5 years; and $48 \%$ in the vaccinated between 5 and 6 years.

Table 1 - Proportion of individuals with protective antibody titers $(r S B A \geq 8) 6$ years after vaccination with MCC vaccines

\begin{tabular}{cc}
\hline $\begin{array}{c}\text { Age at primary } \\
\text { immunization }\end{array}$ & $\begin{array}{c}\text { Individuals with } \\
\text { protective antibody } \\
\text { titers (rSBA } \geq \mathbf{8})\end{array}$ \\
\hline$<6$ months & $12 \%$ \\
5 to 11 months & $16 \%$ \\
12 to 23 months & $26 \%$ \\
2 to 3 years & $23 \%$ \\
3 to 4 years & $33 \%$ \\
4 to 5 years & $26 \%$ \\
5 to 7 years & $48 \%$ \\
\hline
\end{tabular}

rSBA = serum bactericidal antibody measured with rabbit complement Adapted from Perret et al. ${ }^{42}$

In contrast, children that had been vaccinated at older ages, between 6-15 years, maintained high rates of persistence of protective antibody titers ( 4 to 5 years after receiving the vaccine, $79 \%$ of the immunized children between $6-9$ years and $88 \%$ of the immunized between 10-15 years maintained $r S B A \geq 8) .43$ These findings of prolonged persistence of antibody titers, when the vaccine was performed in children above 5 years old, were reproduced in a similar study performed in the Netherlands. 36,37 These data confirm that the immune response provided by the MCC vaccines is age-dependent, i.e., subjects vaccinated at older ages present more consistent and longer lasting responses. This recent evidence of rapid loss of protective antibody titers for children immunized in the first 6 years of life suggests that approximately $75 \%$ of these children are susceptible to the risk of carriage and to developing the disease when they enter adolescence.

The routine immunization scheme in Brazil currently licensed for the MCC vaccines consists of two doses, and can be initiated from 2 months (Novartis MCC-CRM197 and Baxter MCC-TT) or from 3 months old (Wyeth MCC-CRM197), with a booster dose at 12 months old. For children above 1 year old, adolescents and adults, any of the three vaccines should be used in a single dose.

\section{New vaccines}

In 2005, it was licensed in the U.S. and later in other countries a quadrivalent meningococcal vaccine (Sanofi Pasteur), based on safety and immunogenicity data, containing $4 \mu \mathrm{g}$ of the polysaccharides A, C, W135 and $Y$ conjugated to $48 \mu \mathrm{g}$ of diphtheria toxoid protein. Recently, it was licensed in several countries, including Brazil, another quadrivalent meningococcal conjugate vaccine (Novartis) containing $10 \mu \mathrm{g}$ of the polysaccharide $A$ and $5 \mu \mathrm{g}$ of the polysaccharides C, W135 and Y conjugated to CRM197. A third quadrivalent meningococcal vaccine conjugated to tetanus toxoid (GSK) is in its final development stages. The Advisory Committee for the Immunization Practices currently recommends, in the U.S., the quadrivalent meningococcal conjugate vaccine to all adolescents from 11 to 12 years, with a booster dose after 5 years. Adolescents from 13 to 18 years, not previously vaccinated, should also be vaccinated. 44

In Canada, recent data showing that the immune response triggered by the $\mathrm{MCC}$ vaccines in children have limited duration, encouraged the recommendation of one dose of the quadrivalent meningococcal conjugate vaccine at 12 years old to guarantee the continued protection against the disease during adolescence and throughout young adulthood, extending the protection to other serogroups included in the vaccines. 45,46

\section{Conclusions}

The experience gained over 10 years after the introduction of MCC vaccines in immunization programs in Europe, Australia and North America27-45 allowed new evidence to be incorporated regarding the characteristics of the immune response induced by these vaccines, with clear implications in the recommendations as how they should be used in different epidemiologic scenarios. Among these important observations, the relation of the immune response, after primary immunization or after booster dose, with the age at which the vaccine is administered should be highlighted. Infants and children younger than 6 years, probably due to immaturity of the immune system and lack of opportunities of exposure of the immune system to the species of meningococci, present a immune response of shorter duration, with absence of circulating protective antibodies few years after the vaccination. Besides, the occurrence of meningococcal disease cases in previously vaccinated children showed that the presence of immunologic memory, due to the possibility of short periods of incubation 
of the disease, is not always sufficient to provide protection for individuals when exposed to the bacteria. In adolescents, however, the MCC vaccines proved to be associated to a robust immune response, with persistence of protective antibody titers for an extended period, ensuring, thus, the protection of a significant proportion of these vaccinated adolescents until adulthood, when a significant decrease in incidence rates is observed in endemic conditions.

We also point that, despite the seroprevalence studies conducted in the UK and the Netherlands demonstrate that a significant portion of the population (those who were vaccinated as children) is in this moment susceptible to the meningococcal disease, the effect exerted by the herd immunity, achieved by vaccination of age groups responsible for high rates of meningococcal carriage in nasopharynx - particularly the adolescents in the case of the European countries -, led to a substantial decrease in the circulation of the $\mathrm{C}$ meningococcus, preserving, thus, the population protected.

The protection granted by MCC vaccines in populations undergoing mass immunization proved to be, thus, dependent on a combination of maintenance of bactericidal antibody titers, presence of immunologic memory and, especially, the induction of herd immunity.

In Brazil, until October 2010, the MCC vaccines were used only in private clinics, for the control of outbreaks and also for high-risk patients at the Referred Center for Special Immune-biological Products. From late 2010, MCC vaccine was introduced into the routine immunization schedule of infants, in two doses, at 3 and 5 months old, with a booster dose at 12 months of age, not having so far included children over 2 years old or adolescents.

The recent findings regarding the absence of protective antibody titers few years after vaccination in infants and young children and the limitation of immunologic memory in maintaining them protected in the absence of circulating antibody titers associated to protection, in a scenario such as the one we have in the country at the moment, where the occurrence of the important effect of herd protection is not expected, emphasize the need for the adoption of an immunization scheme appropriate to our reality, with the aim to guarantee individual protection against meningococcal disease throughout childhood and adolescence. These recommendations should be adapted, over time, to the current vaccination schedules of the Brazilian Society of Pediatrics and the Brazilian Society of Immunizations. Therefore:

- For children who received vaccination in the first 2 years of life, include an additional dose, between 5 and 6 years old, and another booster dose at 11 years old, with a minimal interval of 5 years in relation to the last dose received, to guarantee individual protection of adolescents in our location, where the effect of the herd protection does not exist at the moment. This additional dose at 11 years old, considering the epidemiology of the disease, may be done with one of the tetravalent meningococcal conjugate vaccines, to extend protection to other serogroups included in the vaccine $(A, Y$ and W135).

- For children who were vaccinated between 2 and 5 years, include an additional dose of MCC vaccine after 5 years of the last dose received and another booster dose, preferably with a tetravalent conjugate vaccine, 5 years later.

- For children vaccinated between 6 and 10 years, include a booster dose, 5 years later, preferably with a tetravalent conjugate vaccine.

- For children, adolescents and adults of the so-called risk groups for developing meningococcal disease (complement deficiency and asplenic), the practice of administering the MCC vaccine at regular intervals of 5 years should be recommended while these individuals remain at increased risk of developing the disease.

Finally, we emphasize that the incorporation to the National Immunization Program of the MCC vaccine in the routine of children younger than 2 years old, which is the age group with the highest incidence rates of the disease in Brazil, anticipates an immediate impact, with significant reduction of morbidity and mortality. In the moment when we observe the effect of herd immunity in our population, what will probably only occur with the expansion of the immunization to other age groups, the need to maintain booster doses for individual protection of children and adolescents may be reviewed.

\section{Acknowledgements}

To Dr. José Geraldo Leite Ribeiro, Professor of the School of Medical Sciences of Minas Gerais, and to Dr. Renato de Ávila Kfouri, pediatrician at the Hospital e Maternidade Santa Joana, for the important suggestions and critics throughout the present research.

\section{References}

1. Pan American Health Organization. Situación actual de la vigilancia de la enfermedad meningocócica en la región de las Américas. Washington, DC: PAHO; 2007. p. 2-23. CDC-PAHO/ $\mathrm{Dpc} / \mathrm{Cd} / \mathrm{A} / 482$.

2. Granoff DM, Harrison LH, Borrow R. Meningococcal vaccines. In: Plotkin SA, Orenstein WA, Offit PA, editors. Vaccines. 5th ed. Philadelphia: Saunders/Elsevier; 2008. p. 399-434

3. Miller E, Salisbury D, Ramsay M. Planning, registration, and implementation of an immunisation campaign against meningococcal serogroup $C$ disease in the UK: a success story. Vaccine. 2001;20:S58-67.

4. Trotter CL, Andrews NJ, Kaczmarski EB, Miller E, Ramsay ME. Effectiveness of meningococcal serogroup $C$ conjugate vaccine 4 years after introduction. Lancet. 2004;364:365-7.

5. American Academy of Pediatrics. Meningococcal infections. In: Pickering LK, Baker CI, Kimberlin DW, Long SS, editors. Red Book: 2009 Report of the Committee on Infectious Diseases. 28th ed. Elk Grove Village, IL: American Academy of Pediatrics; 2009. p. 455-63. 
6. Sáfadi MA, Barros AP. Meningococcal conjugate vaccines: efficacy and new combinations. J Pediatr (Rio J). 2006;82:S35-44.

7. Bilukha OO, Rosenstein N; National Center for Infectious Diseases, Centers for Disease Control and Prevention (CDC). Prevention and control of meningococcal disease. Recommendations of the Advisory Committee on Immunization Practices (ACIP). MMWR Recomm Rep. 2005;54:1-21.

8. Balmer $\mathrm{P}$, Borrow R, Miller E. Impact of meningococcal $\mathrm{C}$ conjugate vaccine in the UK. J Med Microbiol. 2002;51:717-22.

9. Danzig L. Meningococcal vaccines. Pediatr Infect Dis J. 2004;23:S285-92

10. Trotter CL, Maiden MC. Meningococcal vaccines and herd immunity: lessons learned from serogroup C conjugate vaccination programs. Expert Rev Vaccines. 2009;8:851-61.

11. Stephens DS. Uncloaking the meningococcus: dynamics of carriage and disease. Lancet. 1999;353:941-2.

12. Goldschneider I, Gotschlich EC, Artenstein MS. Human immunity to the meningococcus. II. Development of natural immunity. J Exp Med. 1969;129:1327-48.

13. Hankins J. Toward high quality medical care for sickle cell disease: are we there yet? J Pediatr (Rio J). 2010;86:256-8.

14. Harrison LH, Trotter CL, Ramsay ME. Global epidemiology of meningococcal disease. Vaccine. 2009;27:B51-63.

15. Pollard AJ, Maiden MC. Epidemic meningococcal disease in subSaharan Africa - towards a sustainable solution? Lancet Infect Dis. $2003 ; 3: 68-70$.

16. Meningococcal vaccines: polysaccharide and polysaccharide conjugate vaccines. Wkly Epidemiol Rec. 2002;77:331-9.

17. Cohn AC, MacNeil JR, Harrison LH, Hatcher C, Theodore J, Schmidt $M$, et al. Changes in Neisseria meningitidis disease epidemiology in the United States, 1998-2007: implications for prevention of meningococcal disease. Clin Infect Dis. 2010;50:184-91.

18. Brasil. Ministério da Saúde. Secretaria de Vigilância em Saúde. Meningites. In: Guia de vigilância epidemiológica. $6^{a}$ ed. Brasília: Ministério da Saúde; 2005. p. 541-69.

19. Sacchi CT, Fukasawa LO, Gonçalves MG, Salgado MM, Shutt KA, Carvalhanas TR, et al. Incorporation of real-time PCR into routine public health surveillance of culture negative bacterial meningitis in São Paulo, Brazil. PLoS One. 2011;6:e20675.

20. Sáfadi MA, Cintra OA. Epidemiology of meningococcal disease in Latin America: current situation and opportunities for prevention. Neurol Res. 2010;32:263-71.

21. São Paulo. Secretaria de Saúde do Estado de São Paulo. Centro de Vigilância Epidemiológica "Prof. Alexandre Vranjac". Respiratory transmitted diseases. http://www.cve.saude.sp.gov.br/htm/resp/ dm_soro.htm. Access:01/07/2011.

22. Iser BP, Lima HC, De Moraes C, De Almeida RP, Watanabe LT, Alves $\mathrm{SL}$, et al. Outbreak of Neisseria meningitidis $C$ in workers at a large food-processing plant in Brazil: challenges of controlling disease spread to the larger community. Epidemiol Infect. 2011:1-10. [Epub ahead of print]

23. de Lemos AP, Yara TY, Gorla MC, de Paiva MV, de Souza AL, Gonçalves MI, et al. Clonal distribution of invasive Neisseria meningitidis serogroup C strains circulating from 1976 to 2005 in greater São Paulo, Brazil. J Clin Microbiol. 2007;45:1266-73.

24. Weidlich L, Baethgen LF, Mayer LW, Moraes C, Klein CC, Nunes LS, et al. High prevalence of Neisseria meningitidis hypervirulent lineages and emergence of W135:P1.5,2:ST-11 clone in Southern Brazil. J Infect. 2008;57:324-31.

25. Barroso DE, Rebelo MC. Recognition of the epidemiological significance of Neisseria meningitidis capsular serogroup W135 in the Rio de Janeiro region, Brazil. Mem Inst Oswaldo Cruz. 2007; 102:773-5.

26. Lemos AP, Harrison LH, Lenser M, Sacchi CT. Phenotypic and molecular characterization of invasive serogroup W135 Neisseria meningitidis strains from 1990 to 2005 in Brazil. J Infect. 2010;60:209-17.

27. Balmer $P$, Borrow R, Miller E. Impact of meningococcal $C$ conjugate vaccine in the UK. J Med Microbiol. 2002;51:717-22.

28. Ramsay ME, Andrews NJ, Trotter CL, Kaczmarski EB, Miller E. Herd immunity from meningococcal serogroup $C$ conjugate vaccination in England: database analysis. BMJ. 2003;326:365-6.

29. Maiden MC, Stuart JM; UK Meningococcal Carraige Group. Carriage of serogroup $\mathrm{C}$ meningococci 1 year after meningococcal $\mathrm{C}$ conjugate polysaccharide vaccination. Lancet. 2002;359:1829-31.

30. Salleras $L$, Domínguez A, Cardeñosa N. Impact of mass vaccination with polysaccharide conjugate vaccine against serogroup C meningococcal disease in Spain. Vaccine. 2003;21:725-8.

31. Cano R, Larrauri A, Mateo $S$, Alcalá B, Salcedo C, Vázquez JA. Impact of the meningococcal $C$ conjugate vaccine in Spain: an epidemiological and microbiological decision. Euro Surveill. 2004;9:11-5

32. Auckland C, Gray S, Borrow R, Andrews N, Goldblatt D, Ramsay $M$, et al. Clinical and immunologic risk factors for meningococcal $\mathrm{C}$ conjugate vaccine failure in the United Kingdom. J Infect Dis. 2006; 194:1745-52.

33. United Kingdom, Department of Health. Planned changes to the routine Childhood Immunisation Programme. 2006. http:// www.dh.gov.uk/en/Publicationsandstatistics/Lettersandcirculars/ Dearcolleagueletters/DH_4128120. Acesso: 01/10/2010.

34. Campbell H, Andrews N, Borrow R, Trotter C, Miller E. Updated postlicensure surveillance of the meningococcal $C$ conjugate vaccine in England and Wales: effectiveness, validation of serological correlates of protection, and modeling predictions of the duration of herd immunity. Clin Vaccine Immunol. 2010;17:840-7.

35. de Greeff SC, de Melker HE, Spanjaard L, Schouls LM, van Derende A. Protection from routine vaccination at the age of 14 months with meningococcal serogroup C conjugate vaccine in the Netherlands. Pediatr Infect Dis J. 2006;25:79-80.

36. de Voer RM, Mollema L, Schepp RM, de Greeff SC, van Gageldonk PG, de Melker HE, et al. Immunity against Neisseria meningitidis serogroup $\mathrm{C}$ in the Dutch population before and after introduction of the meningococcal c conjugate vaccine. PLoS One. 2010;5:e12144.

37. de Voer RM, van der Klis FR, Niers LE, Rijkers GT, Berbers GA. Absence of Neisseria meningitidis serogroup C-specific antibodies during the first year of life in the Netherlands: an age group at risk? Clin Vaccine Immunol. 2009;16:1521-3.

38. Kafetzis DA, Stamboulidis KN, Tzanakaki G, Kourea Kremastinou J, Skevaki CL, Konstantopoulos A, et al. Meningococcal group C disease in Greece during 1993-2006: the impact of an unofficial single-dose vaccination scheme adopted by most paediatricians. Clin Microbiol Infect. 2007;13:550-2.

39. Trotter CL, Ramsay ME. Vaccination against meningococcal disease in Europe: review and recommendations for the use of conjugate vaccines. FEMS Microbiol Rev. 2007;31:101-7.

40. Larrauri A, Cano R, García M, Mateo S. Impact and effectiveness of meningococcal $C$ conjugate vaccine following its introduction in Spain. Vaccine. 2005;23:4097-100.

41. De Wals P, Deceuninck G, Boulianne N, De Serres G. Effectiveness of a mass immunization campaign using serogroup $C$ meningococcal conjugate vaccine. JAMA. 2004;292:2491-4.

42. Perrett KP, Winter AP, Kibwana E, Jin C, John TM, Yu LM, et al. Antibody persistence after serogroup $C$ meningococcal conjugate immunization of United Kingdom primary-school children in 19992000 and response to a booster: a phase 4 clinical trial. Clin Infect Dis. 2010;50:1601-10.

43. Snape MD, Kelly DF, Lewis S, Banner C, Kibwana L, Moore CE, et al. Seroprotection against serogroup $C$ meningococcal disease in adolescents in the United Kingdom: observational study. BMJ. 2008; 336:1487-91.

44. Centers for Disease Control and Prevention (CDC). Updated recommendations for use of meningococcal conjugate vaccines: Advisory Committee on Immunization Practices (ACIP), 2010. MMWR Morb Mortal Wkly Rep. 2011;60:72-6.

45. National Advisory Committee on Immunization (NACI). An update on the invasive meningococcal disease and meningococcal vaccine conjugate recommendations. An Advisory Committee Statement (ACS). Can Commun Dis Rep. 2009;35:1-40.

46. De Wals P, Deceuninck G, Lefebvre B, Boulianne N, De Serres G. Effectiveness of serogroup $C$ meningococcal conjugate vaccine: a 7-year follow-up in Quebec, Canada. Pediatr Infect Dis J. $2011 ; 30: 566-9$

Correspondence:

Marco Aurélio Palazzi Sáfadi

Alameda dos Indígenas, 228

CEP 04059-060 - São Paulo, SP - Brazil

Tel.: + 55 (11) 3053.3005

Fax: +55 (11) 5594.7579

E-mail: masafadi@uol.com.br 